Draft VERSion SEPTEMBer 25, 2018

Preprint typeset using LATEX style AASTeX6 v. 1.0

\title{
CONVECTIVELY DRIVEN SINKS AND MAGNETIC FIELDS IN THE QUIET SUN
}

\author{
Iker S. Requerey ${ }^{1,2,3}$, Jose Carlos Del Toro Iniesta ${ }^{1}$, Luis R. Bellot Rubio ${ }^{1}$, Valentín Martínez Pillet ${ }^{4}$, \\ Sami K. Solanki ${ }^{5,6}$, And Wolfgang Schmidt $^{7}$ \\ ${ }^{1}$ Instituto de Astrofísica de Andalucía (CSIC), Apdo. de Correos 3004, E-18080 Granada, Spain \\ ${ }^{2}$ Instituto de Astrofísica de Canarias, Vía Láctea s/n, E-38205 La Laguna, Tenerife, Spain \\ ${ }^{3}$ Departamento de Astrofísica, Universidad de La Laguna, E-38206 La Laguna, Tenerife, Spain \\ ${ }^{4}$ National Solar Observatory, 3665 Discovery Drive, Boulder, CO 80303, USA \\ ${ }^{5}$ Max-Planck-Institut für Sonnensystemforschung, Justus-von-Liebig-Weg 3, 37077 Göttingen, Germany \\ ${ }^{6}$ School of Space Research, Kyung Hee University, Yongin, Gyeonggi, 446-701, Republic of Korea \\ ${ }^{7}$ Kiepenheuer-Institut für Sonnenphysik, Schöneckstr. 6, 79104 Freiburg, Germany
}

\begin{abstract}
We study the relation between mesogranular flows, convectively driven sinks and magnetic fields using high spatial resolution spectropolarimetric data acquired with the Imaging Magnetograph eXperiment on board SUNRISE. We obtain the horizontal velocity flow fields of two quiet-Sun regions $\left(31.2 \times 31.2 \mathrm{Mm}^{2}\right)$ via local correlation tracking. Mesogranular lanes and the central position of sinks are identified using Lagrange tracers. We find $6.7 \times 10^{-2}$ sinks per $\mathrm{Mm}^{2}$ in the two observed regions. The sinks are located at the mesogranular vertices and turn out to be associated with (1) horizontal velocity flows converging to a central point and (2) long-lived downdrafts. The spatial distribution of magnetic fields in the quiet Sun is also examined. The strongest magnetic fields are preferentially located at sinks. We find that $40 \%$ of the pixels with longitudinal component of the magnetic field stronger than $500 \mathrm{G}$ are located in the close neighborhood of sinks. In contrast, the small-scale magnetic loops detected by Martínez González et al. in the same two observed areas do not show any preferential distribution at mesogranular scales. The study of individual examples reveals that sinks can play an important role in the evolution of quiet-Sun magnetic features.
\end{abstract}

Keywords: Sun: granulation - Sun: magnetic fields - Sun: photosphere - methods: observational techniques: polarimetric

\section{INTRODUCTION}

Most quiet-Sun magnetic fields evolve on the solar surface driven by convective motions. The largest magnetic structures outline the boundaries of supergranular cells - the magnetic network. Inside the supergranular cells smaller magnetic flux concentrations of both polarities permeate the solar internetwork. They tend to concentrate in mesogranular lanes (Yelles Chaouche et al. 2011) and a significant fraction of the magnetic flux emerges into the surface co-spatially with granules in the form of smallscale magnetic loops (Martínez González et al. 2007; Centeno et al. 2007; Martínez González \& Bellot Rubio 2009; Martínez González et al. 2010; Danilovic et al. 2010a; Martínez González et al. 2012).

Convection displays highly localized downdrafts where cold plasma returns to the solar interior (Spruit et al.

iker@iac.es
1990; Stein \& Nordlund 1998; Nordlund et al. 2009). Due to conservation of the angular momentum, a vortex can be formed as the plasma approaches the downdraft (the bathtub effect, Nordlund 1985). In fact, small-scale vortex flows are ubiquitous in simulations of solar surface convection (e.g., Kitiashvili et al. 2011; Shelyag et al. 2011; Moll et al. 2011; Steiner \& Rezaei 2012). The plasma can also drag magnetic fields toward the draining point, where they can be intensified up to $\mathrm{kG}$ values (Danilovic et al. 2010b; Kitiashvili et al. 2010). As a consequence, a large amount of vorticity can be generated through the interaction of plasma and magnetic fields in the intergranular juctions (Shelyag et al. 2011). Vertical vorticity is known to concentrate preferentially in negative divergence areas, i.e., downflow regions (Wang et al. 1995; Pötzi \& Brandt 2005, 2007). Vortex flows are observed at large scales (up to $20 \mathrm{Mm}$, Brandt et al. 1988; Attie et al. 2009) in supergranular junctions and at smaller scales $(\lesssim 0.5 \mathrm{Mm}$, Bonet et al. 2008, 2010; Vargas Domínguez et al. 2011) in granular ones. Small-scale whirlpools are also visible in the chro- 
mosphere (Wedemeyer-Böhm \& Rouppe van der Voort 2009; Park et al. 2016) and their imprints have been identified in the transition region and low corona (Wedemeyer-Böhm et al. 2012).

Vortex flows harboring magnetic fields are rather abundant (Bonet et al. 2010). In particular, Balmaceda et al. (2010) showed evidence of smallscale magnetic concentrations being dragged toward the center of a convergence flow point. The same event was further studied by Vargas Domínguez et al. (2015), who found sudden downflows and intensification processes of the magnetic concentrations. Magnetic field enhancements preceded by strong downdrafts are generally understood in the context of convective collapse (Parker 1978; Webb \& Roberts 1978; Spruit 1979; Spruit \& Zweibel 1979). Manso Sainz et al. (2011) found that the trajectories of some small-scale loop footpoints describe a vortical motion suggesting that they may be engulfed by a downdraft. Recently, Requerey et al. (2014) observed a loop footpoint being advected and concentrated in a point-like sink together with other same-polarity weak magnetic patches. All these papers, and the previous knowledge that intergranular magnetic fields tend to concentrate near the intersections of multiple granules, strengthen the idea that localized downdrafts ${ }^{1}$ are places where the concentration of magnetic fields is favored. In order to confirm such a relation, quantitative and statistical information is still required.

The aim of this work is to provide a quantitative basis for the association between convectively driven point-like sinks and small-scale magnetic fields in the quiet Sun. We use spectropolarimetric observations from the Imaging Magnetograph eXperiment (IMaX; Martínez Pillet et al. 2011) on board the SUNRISE balloon-borne solar observatory (Solanki et al. 2010; Barthol et al. 2011; Berkefeld et al. 2011; Gandorfer et al. 2011). IMaX provides stable time series of both intensity and polarization filtergrams at high spatial resolution $(\sim 100 \mathrm{~km})$. Using the same SuNRISE/IMaX data, Yelles Chaouche et al. (2011) clearly demonstrated that magnetic elements are preferentially located in mesogranular lanes and Bonet et al. (2010) observed a large number of magnetic features swirling in convectively driven vortex flows. In the latter work, they detected vortices from the horizontal velocities obtained through local correlation tracking (LCT) of magnetograms and other IMaX images. They found a mean vortex duration of about 8 minutes and several events appearing at the same location in the course of the time

\footnotetext{
${ }^{1}$ We shall use the term sink throughout the paper as a synonym of these localized downdrafts.
}

series. The recurrent events were also observed in the flow field maps when averaged over the entire data set. In the present paper we find that such long-lived sinks are located in mesogranular junctions and that they are places where the strongest magnetic fields tend to concentrate.

\section{OBSERVATIONS}

We use high-quality spectropolarimetric data obtained with IMaX on board the SUNRISE balloon-borne solar observatory. IMaX is a dual-beam imaging spectropolarimeter with full Stokes vector capabilities in the Fe I line at $525.02 \mathrm{~nm}$ (Landé factor $\mathrm{g}=3$ ). The line is sampled by a Fabry-Pérot interferometer at five wavelength positions located at $\lambda=-8,-4,+4,+8$, and +22.7 pm from the line center (V5-6 mode of IMaX; see Martínez Pillet et al. 2011, for details). The polarization analysis is performed by two liquid crystal variable retarders and a beam splitter. The image sequences were recorded close to a quiet area at disk center on 2009, June 9. We analyze two different time series, namely, $S_{1}$, from 00:36:03 to 00:58:46 UT (22.7 minutes duration) and $S_{2}$, from 01:30:54 to 02:02:29 UT (32.1 minutes), with a cadence of $33.25 \mathrm{~s}$, and a spatial sampling of $39.9 \mathrm{~km}$.

The science images were reconstructed using phase diversity measurements (Gonsalves 1982; Paxman et al. 1996) as described by Martínez Pillet et al. (2011). This procedure effectively reduces the IMaX field of view (FOV) down to about $43^{\prime \prime} \times 43^{\prime \prime}\left(31.2 \times 31.2 \mathrm{Mm}^{2}\right)$. After reconstruction, the spatial resolution has been estimated to be $0.15-0$ "' 18 and the noise level in each Stokes parameter is about $3 \times 10^{-3} I_{\mathrm{c}}$ ( $I_{\mathrm{c}}$ being the continuum intensity). We recover information of the vector magnetic field and line-of-sight (LOS) velocities through inversions of the full Stokes vector using the SIR code (Ruiz Cobo \& del Toro Iniesta 1992) as described in Requerey et al. (2015). Height independent values for the three components of the magnetic field and LOS velocity are assumed. The magnetic filling factor (i.e., the fraction of the pixel filled with magnetic field) is set to unity. From the magnetic field strength $B$ and the inclination $\gamma$ we derive the longitudinal component of the magnetic field (hereafter referred to as the longitudinal magnetogram) $B_{\text {long }}=B \cos \gamma$.

\section{CONVECTIVELY DRIVEN SINKS}

\subsection{Identification of mesolanes}

Mesogranulation is a horizontal cellular flow pattern revealed through the LCT technique when applied to intensity images of the solar granulation (November et al. 1981; Simon et al. 1988; Title et al. 1989; Brandt et al. 1991; Muller et al. 1992; Roudier et al. 1998; Yelles Chaouche et al. 2011). Here 

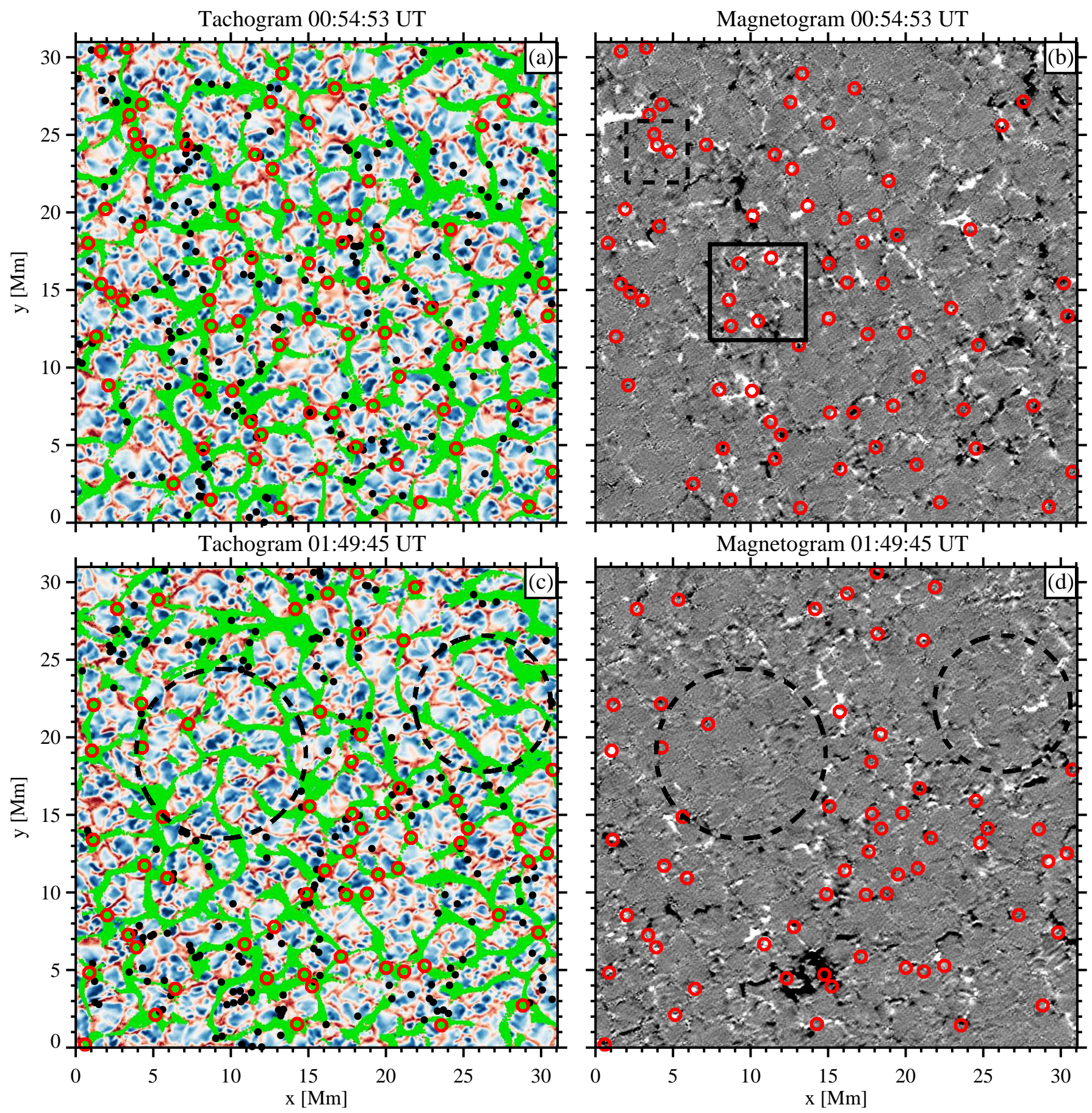

Figure 1. Left panels: green pixels represent locations with more than 2 corks pixel $^{-1}$ at $t=21.6$ minutes and its 1 pixel neighborhoods for time series $\mathrm{S}_{1}$ (a) and $\mathrm{S}_{2}$ (c), respectively. The background image is the LOS velocity at $t=18.3$ minutes (saturated at $\pm 1.5 \mathrm{~km} \mathrm{~s}^{-1}$ ), where red and blue regions correspond to downflow and upflow areas, respectively. Black filled circles show the average position (between the two footpoints) of the small-scale loops detected by Martínez González et al (2012). Right panels: the longitudinal component of the magnetic field, $B \cos \gamma$, at $t=18.3$ minutes (saturated at $\pm 50 \mathrm{G}$ ) for $\mathrm{S}_{1}$ (b) and $\mathrm{S}_{2}(\mathrm{~d})$, respectively. Red circles (with a radius of 9 pixel $\sim 360 \mathrm{~km}$ ) represent the positions of the 131 sinks. The two areas delineated by the dashed circles mark two dead calm regions found by Martínez González et al. (2012). The black dashed box represents the region analyzed in Requerey et al. (2014). The black solid rectangle, with a FOV of $6.2 \times 6.2 \mathrm{Mm}{ }^{2}$, illustrates the area used in Figure 4. 
we search for this pattern in the continuum intensity filtergrams of the two data sets. We use a common time coverage for both time series, namely, the total duration of series $\mathrm{S}_{1}$ (the shorter one), so that we analyze only the first 42 snapshots of $\mathrm{S}_{2}$. This criterion will allow us to equally define inter-mesogranular lanes (called here mesolanes for short) in both time series. We apply a p-mode subsonic filter (Title et al. 1989) to remove the 5 -min solar oscillations. This process degrades the first and the last frames, which are removed from our time series. The final data sets last, therefore, 21.6 minutes. We employ the LCT technique (November \& Simon 1988) as implemented by Molowny-Horas \& Yi (1994) to obtain the mean horizontal velocity field averaged over the duration of the data sets. This technique correlates small local windows in consecutive images to find the best-match displacement. The tracking window is defined by a Gaussian function with a $\mathrm{FWHM}=600 \mathrm{~km}$. After measuring the horizontal velocity vector $\boldsymbol{v}=\boldsymbol{v}_{x}+\boldsymbol{v}_{y}$, we also compute the flow divergence $\nabla \boldsymbol{v}=\frac{\partial \boldsymbol{v}_{x}}{\partial x}+\frac{\partial \boldsymbol{v}_{y}}{\partial y}$ and vertical vorticity $(\nabla \times \boldsymbol{v})_{z}=\frac{\partial \boldsymbol{v}_{y}}{\partial x}-\frac{\partial \boldsymbol{v}_{x}}{\partial y}$.

We outline the location of mesolanes by using Lagrange tracers (corks). Employing the mean horizontal velocity field, we track the evolution of each individual cork as described in Yi (1992). Initially, a cork is located at each pixel of the image. Then the nearest neighbor velocity vector is used to "move" the corks. The trajectories are integrated in time assuming a time step of $33.25 \mathrm{~s}$. For convenience, this value has been chosen to be the same as the cadence of the time series. With this choice the mesogranular pattern is clearly visible after 21.6 minutes (i.e., 39 time steps), when most of the corks have already converged to mesolanes. The thickness and number of mesolanes depends significantly on the time the corks are allowed to move. After 21.6 minutes we find a good compromise between the smallest thickness and maximum number of mesolanes. At that time step we count the number of corks in each pixel to obtain the cork density function, $\rho_{\text {cork }}$ (Yelles Chaouche et al. 2011), and we define the mesolanes as the locations where $\rho_{\text {cork }} \geq 2$, augmented by a 1-pixel wide neighborhood. These areas are marked in Figure 1 (a) and (c) through green pixels overlaid over the LOS velocity maps (tachograms) at $t=18.3$ minutes for time series $\mathrm{S}_{1}$ and $\mathrm{S}_{2}$, respectively. They clearly delineate a fully developed network of mesogranular cells with a size of 5-10 Mm (November et al. 1981).

\subsection{Identification of converging flows}

The mesogranular pattern is visible after only 21.6 minutes. However, if the corks are tracked for even longer, they finally end up (after some 5 hours $^{2}$ ) in well localized points, in places where the horizontal velocity vectors converge on a central point. We obtain therefore the central positions of persistent converging flows, which appear in the mean flow corresponding to the full time series. We identify a total of 131 converging horizontal flows in the two observed regions. Taking into account the spatial area covered by each FOV, we get a density of $6.7 \times 10^{-2}$ converging flows per $\mathrm{Mm}^{2}$.

\subsection{Classification of converging flows}

In previous studies, small whirlpools were first detected as swirling motions of bright points (Bonet et al. 2008). Later they were identified from LCT horizontal velocities of magnetograms (Bonet et al. 2010) and Gband filtergrams (Vargas Domínguez et al. 2011). In the same way as done here, Vargas Domínguez et al. (2011) applied LCT to the whole FOV and duration of two G-band time series (20 minutes each) acquired with the Swedish 1-m solar telescope. By visual inspection of the horizontal flow-field maps, they identified individual vortices as those locations where the horizontal velocity vectors converge to a central point and form a swirl. They found these regions to be coincident with the final destination of corks. However they detected many other places where the corks got accumulated without any apparent swirling motion.

Here we inspect both the horizontal flow maps and the cork movies to analyze all the detected convergence centers. We realize that the corks follow different trajectories on their way to being engulfed by the converging flows. Some converge radially while other trace a spiral path as they fall into the inflow centers. In Figure 2 black arrows display the distribution of horizontal velocities for examples of two types of converging flows. In the left panel, the vectors point radially towards the center of the image, while in the right panel, they display a swirl. We shall hereafter refer to them as radial flows and vortex flows, respectively. We classify all the converging flows into these two categories by visual inspection. They are labeled as vortex flows when a sense of rotation can be ascribed to them from both cork movies and horizontal flow maps. In all other cases, they are designated as radial flows.

\subsection{Statistics of converging flows}

We detect 46 vortex flows (35\% of the total sample of converging flows) and 85 radial flows. These result in densities of $2.4 \times 10^{-2}$ vortex flows per $\mathrm{Mm}^{2}$

\footnotetext{
2 Notice that the duration of the cork movie is unrelated to the length of the observations. The former has been computed by tracking cork trajectories through the mean horizontal velocity field as described in Section 3.1.
} 


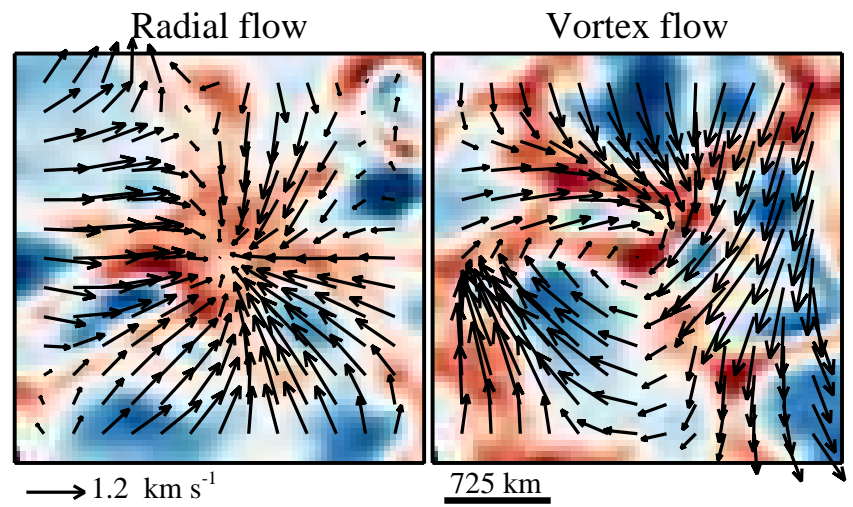

Figure 2. Two examples of converging flows detected in the IMaX data. The background images represent the LOS velocity at $t=18.3$ minutes (saturated at $\pm 1.8 \mathrm{~km} \mathrm{~s}^{-1}$ ). Red and blue regions corresponds to downflow and upflow areas, respectively. The black arrows represent the horizontal velocity vectors obtained from LCT. The left panel shows an example of what we name a radial flow where velocity vectors point radially towards the center of the image. The right panel displays a vortex flow where the velocity vectors exhibit a swirling motion with a clockwise sense of rotation.

and $4.4 \times 10^{-2}$ radial flows per $\mathrm{Mm}^{2}$, respectively. Our values for vortices is comparable to the number obtained by Vargas Domínguez et al. (2011) of 2.8$3.1 \times 10^{-2}$ vortices per $\mathrm{Mm}^{2}$.

In Figure 3 we display histograms of the divergence (left panels) and the vertical vorticity (right panels) at the convergence centers. By construction, all the converging flows (panel a) are located in negative divergence areas with a median value of $-1.9 \times 10^{-3} \mathrm{~s}^{-1}$. The divergence distribution is very similar for both vortex flows (panel e) and radial flows (panel c) with a $25 \%$ smaller median value for the former, suggesting that vortex sinks are $(25 \%)$ less vigorous than uniform ones. Not surprisingly, the distribution of the vertical vorticity is completely different. Panel (d) shows that most of the radial flows have very low vorticity, whereas the negative and positive humps in panel (f) are produced by the distribution of whirls with clockwise and counterclockwise sense of rotation, respectively. ${ }^{3}$ Both clockwise and counterclockwise vortices have a median absolute vertical vorticity of $1.5 \times 10^{-3} \mathrm{~s}^{-1}$. We find a slightly larger number of clockwise $(54 \%)$ than counterclockwise motions (46\%), but the difference is not significant in view of the number of studied events.

\subsection{LOS velocities in converging flows}

If the computed horizontal flows are really describing true mass motions, then the horizontal proper motions should be related to vertical velocities. In partic-

\footnotetext{
${ }^{3}$ Since our classification is visual there is no clear threshold in vertical vorticity that separates vortex flows from radial flows.
}

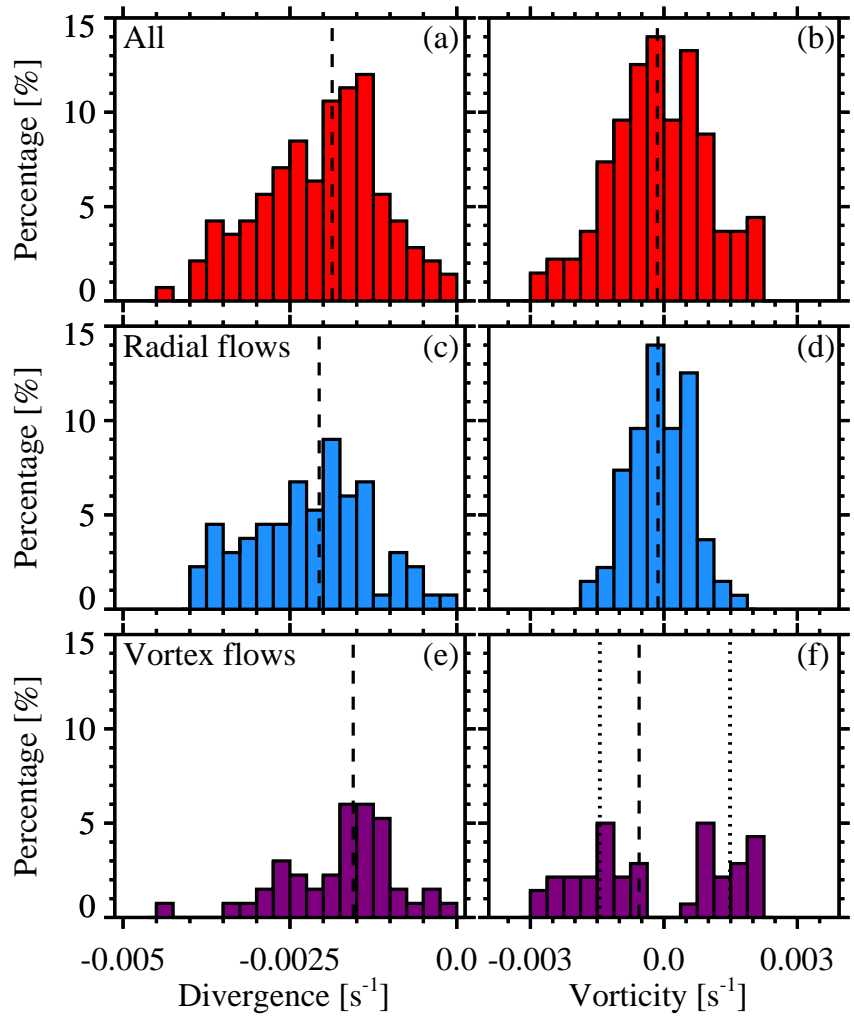

Figure 3. Histograms of divergence (a, c, and e) and vertical vorticity ( $b, d$, and $\mathrm{f}$ ) obtained from LCT proper motions at the convergence centers. Top panels are for all the 131 converging flows, middle panels are for the 85 radial flows, and bottom panels are for the 46 vortex flows. The vertical dashed lines indicate the median values of the distributions. In panel (f) the left and right vertical dotted lines mark the median value of the clockwise and counterclockwise vortices, respectively. We use a bin size of $2.5 \times 10^{-4} \mathrm{~s}^{-1}$ and $3.75 \times$ $10^{-4} \mathrm{~s}^{-1}$ for divergence and vertical vorticity, respectively.

ular, positive and negative divergences (i.e., mesogranules and mesolanes) must be correlated with upflows and downflows, respectively. Equivalently, converging flows should be coincident with downdrafts if they are indeed related to localized sinks.

In previous works (Bonet et al. 2008, 2010; Vargas Domínguez et al. 2011), the authors assumed without further examination that converging flows are locations of sinking gas. Here, we use the capabilities of IMaX to determine the LOS velocities at these locations. For this purpose, we build a binary mask by assuming that the spatial size of a converging flow is given by a 6 pixel $(\sim 240 \mathrm{~km})$ radius circle plus a 3 pixel wide annulus neighborhood. This $240 \mathrm{~km}$ radius corresponds to the mean radius found for converging flows by Vargas Domínguez et al. (2011). In addition, we divide the FOV into mesogranules and mesolanes. The former are defined as those areas in the FOV that are not covered by the latter (defined in Section 3.1). We then compare the histograms of the LOS velocities inferred from the SIR inversions at mesogranules, 

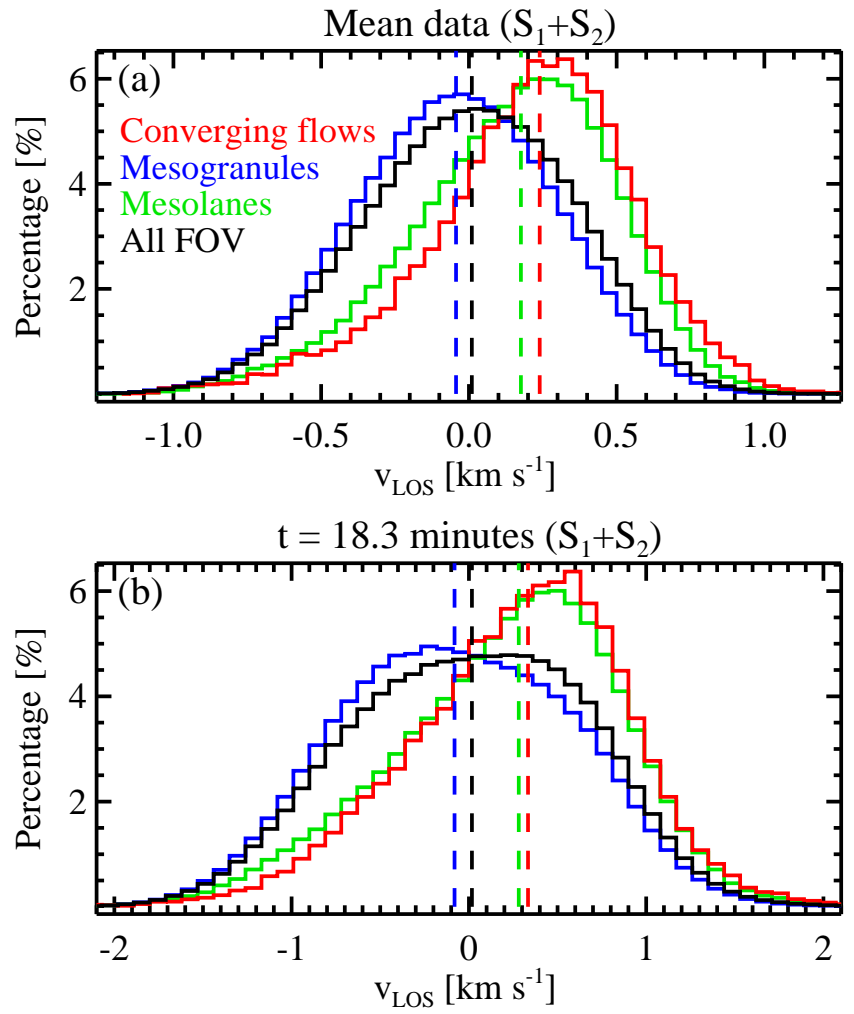

Figure 4. Histograms of the LOS velocity for combined values within both observed areas $\left(\mathrm{S}_{1}+\mathrm{S}_{2}\right)$. Panel (a) plots mean values over the time series, whereas panel (b) shows values within a single snapshots at $t=18.3$ minutes after the start of the time series. Solid lines stand for all pixels in the FOV (black), converging flows (red), mesolanes (green), and mesogranules (blue), respectively. These regions are defined in the text. The corresponding vertical dashed lines indicate the median values of the distributions.

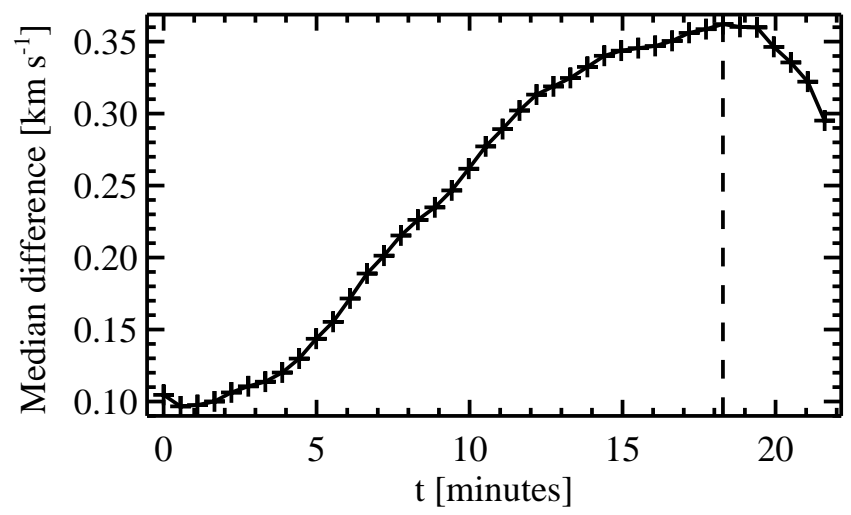

Figure 5. Differences between the LOS velocity median values at mesolanes and mesogranules over the duration of the time series. The vertical dashed line indicates the moment $(t=18.3$ minutes) where the difference is largest.

mesolanes and converging flows with that for the whole FOV.

The position of mesolanes and converging flows depend on the LCT average and therefore their comparison with individual tachograms of the time series is not straightforward. In a first instance, we compare them with the mean LOS velocities (averaged over the duration of the time series) at both observed regions $\left(\mathrm{S}_{1}+\mathrm{S}_{2}\right)$. The resulting histograms are shown in Figure 4 (a). Different line colors indicate different locations, namely, red for converging flows, blue for mesogranules, green for mesolanes, and black for the whole FOV. The corresponding vertical dashed lines indicate the median values of each distribution. Mesogranules are blueshifted and mesolanes redshifted with median values of -0.05 and $0.17 \mathrm{~km} \mathrm{~s}^{-1}$, respectively. The redshift at convergence areas (red line) is even larger than in mesolanes, with a median value of $0.24 \mathrm{~km} \mathrm{~s}^{-1}$. On average, mesogranules appear associated with upflows while mesolanes and converging flows are mostly located at downflows.

If we do the same for individual snapshots of the time series, we find that the correlation between mesolanes and downflows is highest at $t=18.3$ minutes after the start of the time series. This is shown in Figure 5 where we display the differences between the median LOS velocity values at mesolanes and mesogranules over the time series. The vertical dashed line indicates the instant where the difference is largest. The corresponding histograms at $t=18.3$ minutes are shown in Figure 4 (b). The results are similar to those obtained in Figure 4 (a). In this case, mesogranules (blue line), mesolanes (green line) and converging flows (red line) are characterized by median velocities of $-0.1,0.27$, and $0.33 \mathrm{~km} \mathrm{~s}^{-1}$, respectively. Figure 2 shows two representative examples of converging flows associated with downflows.

In summary, we can say that mesolanes and in particular converging flows are (1) preferentially located within intergranular lanes and (2) associated with long-lived downdrafts. Therefore, we have shown that converging flows are indeed locations of sinking gas and we shall hereafter refer to them as convectively driven sinks.

At this point, we examine the question of whether mass is conserved at sinks. For that to occur, the lateral influx of mass over a scale height $H$ should be equal to the vertical mass flux. Approximating the sink geometry by that of a circular cylinder of radius $R$ and height $H$, mass conservation thus requires

$$
2 \pi R H \rho v_{R} \approx \pi R^{2} \rho v_{z} .
$$

To evaluate the lateral and vertical mass fluxes we need the radial velocity at the edge of the sink, $v_{R}$, and the vertical velocity, $v_{z}$. Assuming rotational invariance, the flow divergence can be expressed as $\nabla \boldsymbol{v}=\frac{1}{r} \frac{d\left(r \boldsymbol{v}_{r}\right)}{d r}$ in cylindrical coordinates. Taking $v_{r}=\frac{r}{R} v_{R}$ yields $v_{R}=\frac{R \nabla \boldsymbol{v}}{2}$. With $R=240 \mathrm{~km}$ and $\nabla \boldsymbol{v}=1.9 \times 10^{-3} \mathrm{~s}^{-1}$ (see Section 3.4), we obtain $v_{R}=0.23 \mathrm{~km} \mathrm{~s}^{-1}$. The influx of mass is then $2 \pi R H \rho v_{R}=5.2 \times 10^{19} \rho\left[\mathrm{g} \mathrm{s}^{-1}\right]$ over a density scale height of $150 \mathrm{~km}$ (e.g., Gingerich et al. 
1971). On the other hand, taking $v_{z}=0.33 \mathrm{~km} \mathrm{~s}^{-1}$ yields a vertical mass flux $\pi R^{2} \rho v_{z}=6 \times 10^{19} \rho\left[\mathrm{g} \mathrm{s}^{-1}\right]$, which is in good agreement with the lateral mass influx. Thus, we conclude that mass is approximately conserved in sinks.

\subsection{Spatial distribution of sinks}

In Figure 1 we show the distribution of sinks (red circles) overlaid on the tachograms and longitudinal magnetograms of the two observed areas at $t=18.3$ minutes. Green pixels in the tachograms represent the distribution of corks delineating the mesolanes. The sinks show a mesogranular distribution as most of them are located at the junctions of multiple mesogranular lanes. However, their spatial distribution is not completely uniform and there are some extended areas lacking detected sinks. There are two particularly prominent regions in time series $\mathrm{S}_{2}$. Interestingly they are coincident with the dead calm areas found by Martínez González et al. (2012), characterized by very low magnetic flux and a lack of small-scale magnetic loops. We have marked them with dashed circles in Figures 1 (c) and (d). The two regions contain several mesogranules, but only a single sink is observed in the largest void. Visual inspection of the sinks in the longitudinal magnetograms (Figures $1 \mathrm{~b}$ and d) already reveals that many sinks harbor magnetic fields. This connection between magnetic fields and sinks gets even more evident if one looks at the evolution of magnetic elements. As we will describe in the following Section, magnetic structures are seen to be affected by convectively driven sinks.

\section{THE EVOLUTION OF MAGNETIC FEATURES DRIVEN BY CONVECTIVE MOTIONS}

This Section presents examples of three distinct processes that affect the evolution of quiet-Sun magnetic features: coalescence, cancellation and fragmentation. The statistics of such processes in the SunRISE/IMaX data set has been studied in detail by Anusha et al. (2016). Here we show that these evolutionary processes are driven by convective motions. In particular, coalescence and cancellation of magnetic structures are commonly observed at the sinks. Generally, fragmentation also takes place at sinks. At these locations, however, the resulting fragments are not usually completely detached from each other. Rather, the converging flows tends to merge them again into a single magnetic element. This phenomenon has already been observed by Requerey et al. (2015): multi-cored magnetic structures are seen to live as a single entity for long periods; the individual cores merge and split. Our examples of fragmentation (see Section 4.3) are taken from such multi-cored structures. Outside sinks, the complete splitting of magnetic elements is widely observed when the features are "squeezed" between two converging granules. Note that only illustrative examples are presented and discussed here, whose relevance for processes taking place in other magnetic features will need to be investigated on the basis of a much larger sample.

\subsection{Coalescence}

Figure 6 shows a coalescence process with a selection of three longitudinal magnetograms (upper row) and co-aligned CN-band filtergrams (bottom row). The CN-band filtergrams were acquired with the SunRISE Filter Imager (SuFI; Gandorfer et al. 2011) and have been properly aligned with IMaX images as described in Requerey et al. (2015). The rightmost panel displays the horizontal velocity vectors obtained through the LCT technique averaged over the whole time series of 21.6 minutes (see Section 3). The background image shows the mean tachogram averaged over the same time.

The velocity map reveals a sink as the flows converge towards the center of the image where a downflow is present. In the first magnetogram two weak samepolarity magnetic patches are seen. As time goes by the patches move towards the convergence center where they merge into a single more intense magnetic element. During this coalescence process the longitudinal magnetic field increases from $100 \mathrm{G}$ to about $200 \mathrm{G}$ and a bright point (BP) is also formed as seen in the CN-band filtergrams. Contrary to the standard flux-tube theory (Spruit 1976), we find CN-band BPs related to magnetic elements with fields weaker than $1 \mathrm{kG}$ (see also Section 4.3). Based on a comparison between the same SunRISE observations and MHD simulations, Riethmüller et al. (2014) concluded that all magnetic BPs are indeed associated with $\mathrm{kG}$ fields provided they are spatially resolved at the resolution of SunRISE/IMaX. However, from a simultaneous inversion of near infrared and visible spectral lines with a free magnetic filling factor, Beck et al. (2007) found that the field strength of G-band BPs has a flat distribution from $500 \mathrm{G}$ to $1500 \mathrm{G}$.

\subsection{Cancellation}

Figure 7 shows a partial cancellation process. The bottom row displays the line-core intensity instead of a CN-band filtergram as the event is located outside the FOV of SuFI. The line-core intensity has been determined by fitting a Gaussian to the observed IMaX Stokes $I$ profiles (see Requerey et al. 2015). In the first frame, two diffuse structures of opposite polarities are observed. These structures are concentrated by the horizontal converging flows shown in the velocity map, thus forming two intense magnetic elements that interact with each other (see second frame). At this point, the positive and negative patches have magnetic fluxes of $+6.5 \times 10^{17} \mathrm{Mx}$ and $-9 \times 10^{17} \mathrm{Mx}$, respectively. The positive polarity 


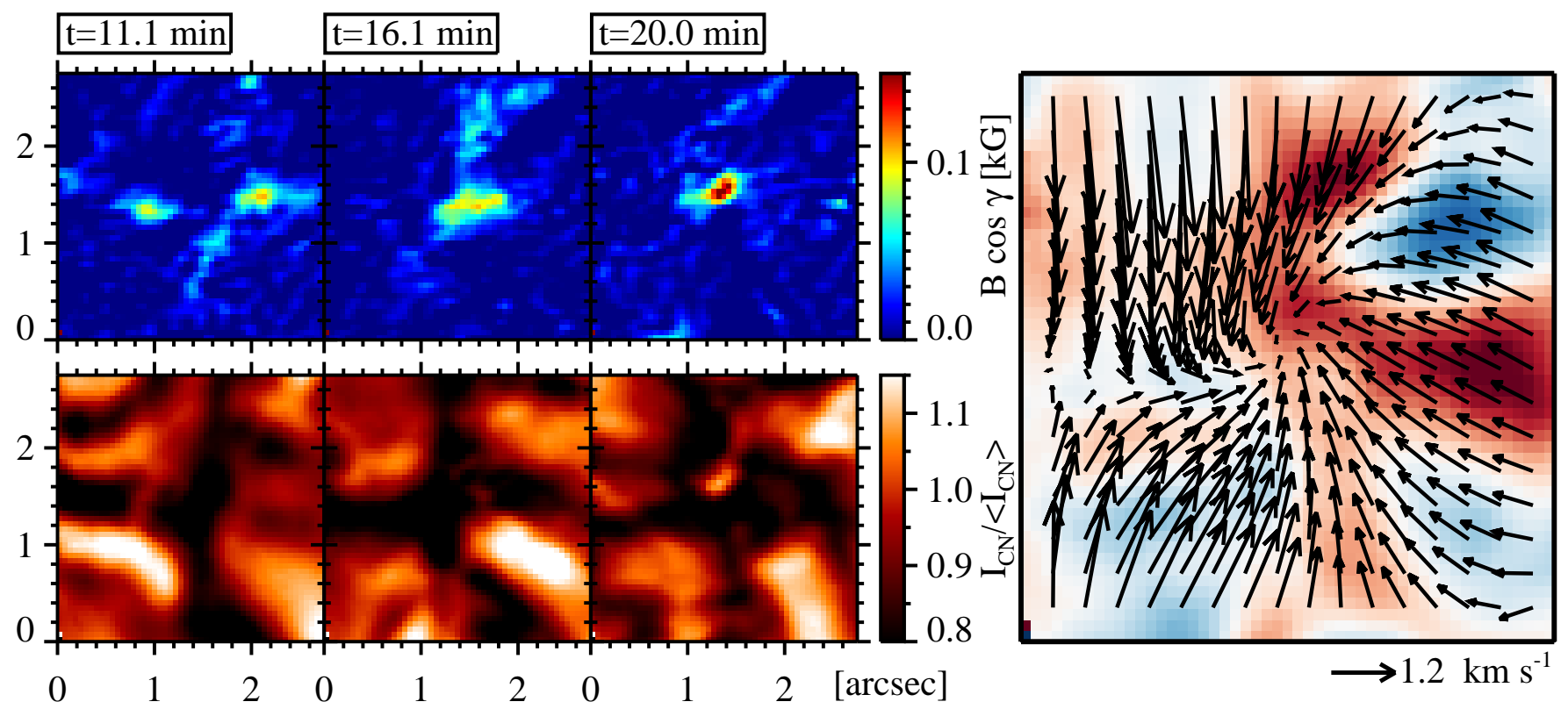

Figure 6. Temporal sequence of a coalescence process. Upper row: IMaX Fe I $525.02 \mathrm{~nm}$ longitudinal magnetic field. Lower row: SuFI images in the CN bandhead, centered at $388 \mathrm{~nm}$ with FWHM $\approx 0.8 \mathrm{~nm}$. The rightmost panel shows the horizontal velocity maps (black arrows) derived through the LCT technique averaged over the whole time series of 21.6 minutes. The background image shows the mean tachogram averaged over the whole time series and saturated at $\pm 0.8 \mathrm{~km} \mathrm{~s}^{-1}$. The black arrows display the horizontal velocity vectors. The length of the black arrow below the lower right corner of the figure corresponds to $1.2 \mathrm{~km} \mathrm{~s}{ }^{-1}$.

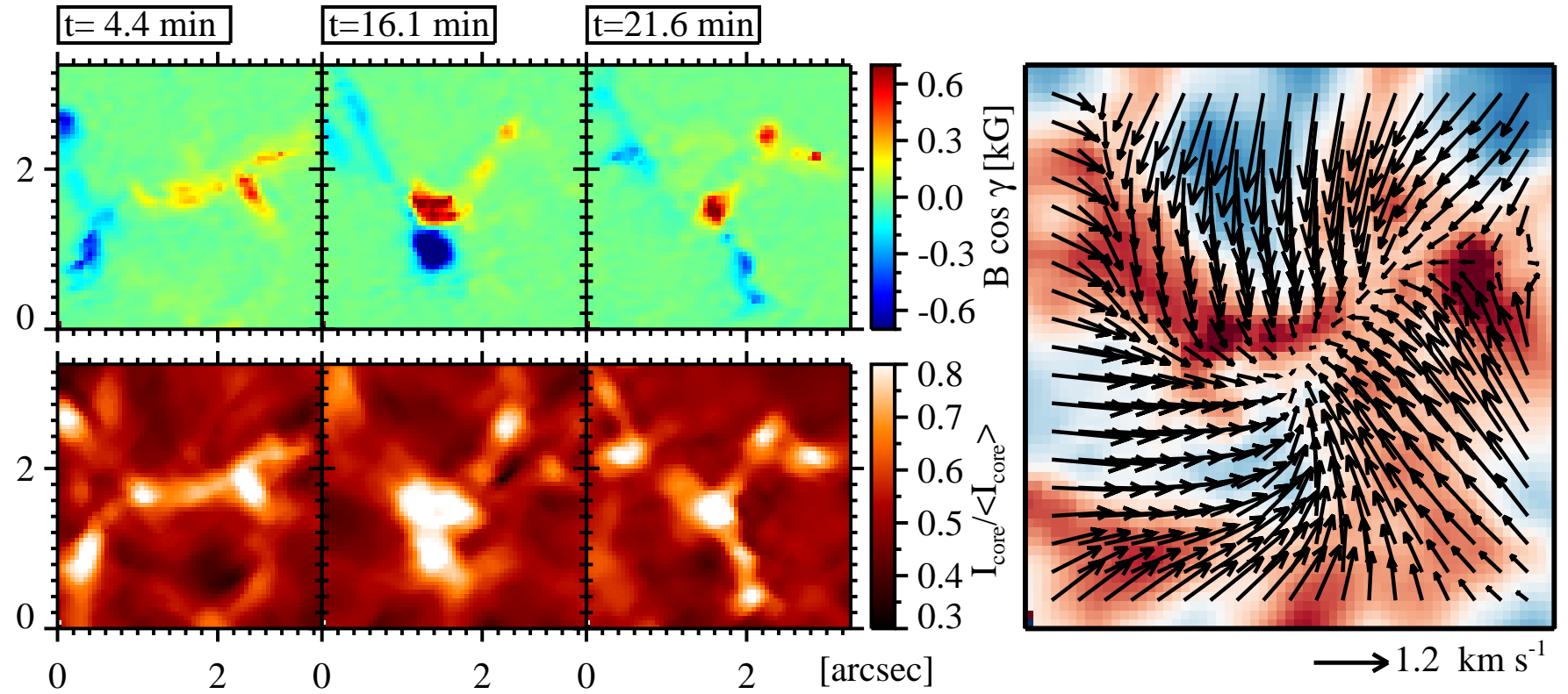

Figure 7. Same as Figure 6, during a partial cancellation process. Here the lower row shows the line-core intensity of the IMaX Fe I line at $525.02 \mathrm{~nm}$ in units of the continuum intensity. Please also note the different color scale in the upper row of images.

feature survives this interaction intact, although with a greatly reduced magnetic flux of $+3.5 \times 10^{17} \mathrm{Mx}$, while the negative one gets more diffuse and decreases its magnetic flux down to $-2.5 \times 10^{17} \mathrm{Mx}$.

\subsection{Fragmentation and merging in a multi-cored} magnetic structure
Figure 8 shows the evolution of a small magnetic feature. It is located within a vortex flow as indicated by the swirl displayed in the horizontal velocity map. In the first image the magnetic feature contains two cores with longitudinal field of $300 \mathrm{G}$ each. Although these cores are likely spatially unresolved, the co-aligned CN-band image confirms that they are indeed separate as each one appears associated with a $\mathrm{CN}$ bright point. This testifies 

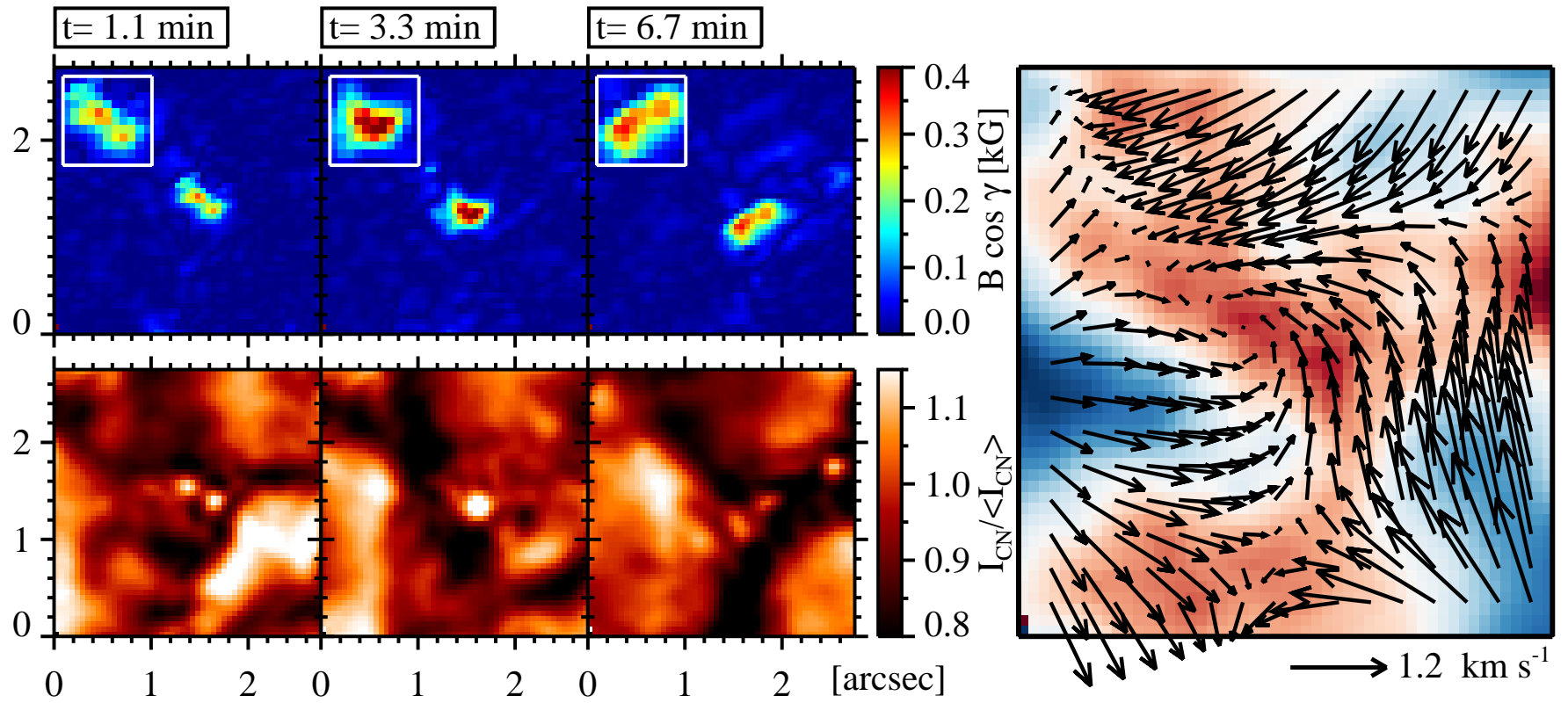

Figure 8. Same as Figure 6, during the evolution of a multi-cored magnetic structure. White boxes in the upper row display a zoom of the magnetic structure.

to the outstanding quality of the SunRISE/IMaX spectropolarimetric capabilities. The time sequence shows that the inner cores, and so the related BPs, merge to form a more intense element, which reaches up to $600 \mathrm{G}$. The coalescence is probably driven by the sink. Soon afterwards, however, the magnetic feature splits again into two weaker components. The evolution of such multi-cored magnetic structures has been studied in detail in Requerey et al. (2015). The continuous merging and splitting processes of the inner cores is governed by the evolution of surrounding granules and results in magnetic field oscillations of the global entity.

\section{CORRELATION BETWEEN MESOGRANULES, SINKS, AND MAGNETIC FIELDS}

From visual inspection of the sinks and the study of individual examples (see also Balmaceda et al. 2010; Bonet et al. 2010; Requerey et al. 2014; Vargas Domínguez et al. 2015), we obtain indications that strong magnetic features concentrate preferentially at well localized places in the centers of sinks. In order to obtain a quantitative estimate of the spatial distribution of magnetic fields ${ }^{4}$, for each threshold value $B_{\text {th }}$ in the range $[0,1000] \mathrm{G}$, we consider the number of pixels with $\left|B_{\text {long }}\right|>B_{\text {th }}$ in the FOV and compute the percentage of those pixels that are located

\footnotetext{
${ }^{4}$ In what follows, we remove from our analysis the prominent positive patch appearing at the top-left edge of the time series $\mathrm{S}_{1}$ (Figure 1 (b)). This magnetic structure enters partially into the FOV in the course of the time series, and it is located at the edge of the FOV, where the horizontal flow fields are not properly determined.
}

within different subareas. The result is displayed in Figure 9 (a) for the combination of both observed regions $\left(\mathrm{S}_{1}+\mathrm{S}_{2}\right)$ at $t=18.3$ minutes. Different line colors indicate different locations, namely, green for mesolanes, blue for mesogranules, and red for sinks. The curves for mesolanes and mesogranules add up to $100 \%$ at each $B_{\text {th }}$ value according to our definition (i.e., the sum of both areas cover the entire FOV), whereas the red curve for sinks is just a subregion of mesolanes. It can be seen that the magnetic structures are mainly located at mesolanes. In agreement with Yelles Chaouche et al. (2011), $80 \%$ of the pixels harboring fields with longitudinal component larger than $100 \mathrm{G}$ are located in mesolanes. We also find that the fraction of pixels stronger than $B_{\mathrm{th}}$ increases linearly with $B_{\text {th }}$ in sinks. Actually, $40 \%$ of the pixels with $\left|B_{\text {long }}\right| \gtrsim 500 \mathrm{G}$ (see vertical dashed line in Figure 9 (a)) are located in the sinks or in their close vicinity. Such a large concentration is very remarkable, since sinks occupy only $3 \%$ of the surface area in contrast with the $26 \%$ of the mesolanes. This statement can be better visualized through the corresponding probability density functions (PDFs) displayed in Figure 9 (b). All the PDFs peak at $0 \mathrm{G}$, and then decrease rapidly toward stronger fields. This decrease is steeper for mesolanes than for sinks, and even more abrupt for mesogranules. All these measurements suggest that the magnetic fields in the quiet-Sun areas are preferentially located at well localized long-lived downdrafts.

On granular scales, observations indicate that there is a continuous injection of magnetic flux in the form of small-scale magnetic loops (e.g., 

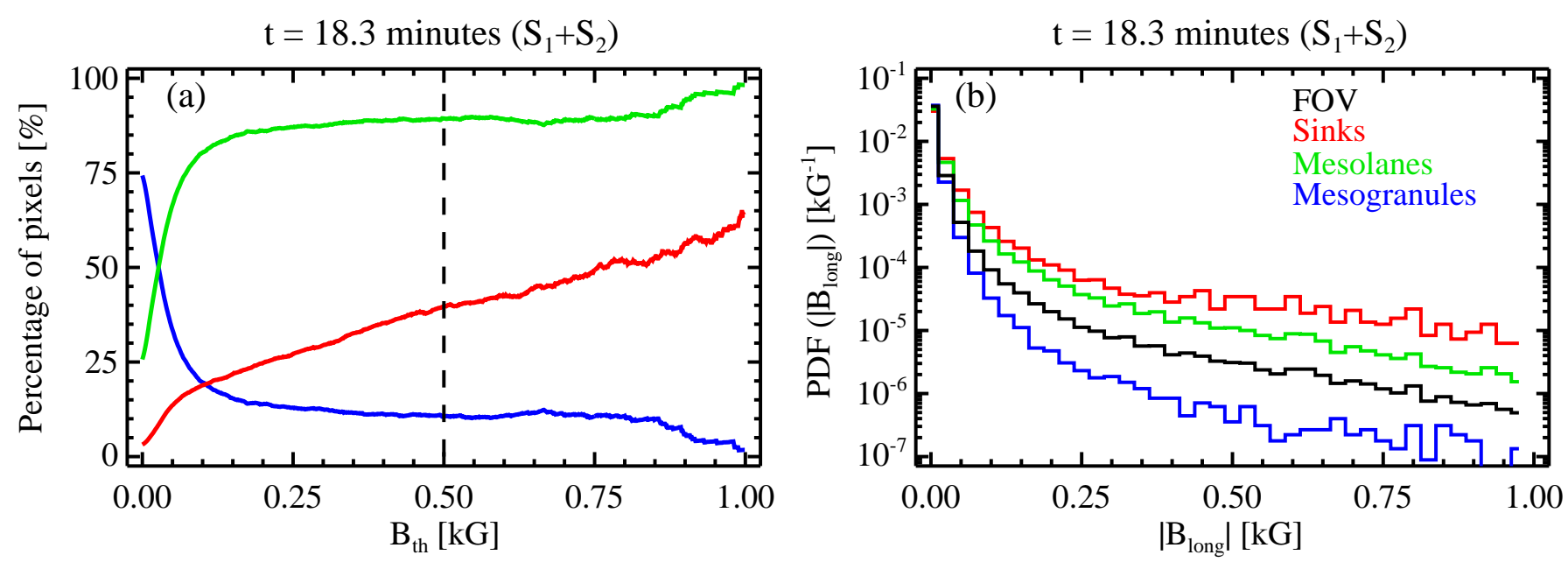

Figure 9. Panels (a): percentage of pixels with $\left|B_{\text {long }}\right|$ above the value $B_{\text {th }}$ given in the abscissa as obtained in both time series at $t=18.3$ minutes. Panel (b): probability density functions of $\left|B_{\text {long }}\right|$ at $t=18.3$ minutes in both time series. The black, red, green, and blue solid lines stand for all pixels in the FOV, sinks, mesolanes, and mesogranules, respectively.

Martínez González \& Bellot Rubio 2009). Exploring, then, whether any preferential location also applies to these loops is in order. In Figure 1 (a) and (c) black filled circles show the distribution of the average position between the footpoints of each detected loop. The locations of loops have been identified by Martínez González et al. (2012). The average initial distance between footpoints was found to be $\sim 0.25 \mathrm{Mm}$. To represent the surface area covered by loops, we have built a binary mask by defining them as $0.25 \mathrm{Mm}$ diameter circles around the average position between both footpoints. We count the number of pixels in both observed regions and find that $69.5 \%$ of them are located in mesogranules, $30.5 \%$ in mesolanes, and only $4.5 \%$ in sinks. These values are close to those of the surface fraction covered by each region $(74 \%$ mesogranules, $26 \%$ mesolanes, and 3\% sinks), suggesting that magnetic loops are homogeneously distributed on mesogranular scales except in the dead calm areas where no loop is seen to emerge (Martínez González et al. 2012).

\subsection{Mesogranule: a case study}

In Figure 10 we zoom in on one of the mesogranules. Panel (a) shows the divergence map with the overlaid white arrows displaying the horizontal flow field. The mesogranule is characterized by a strong positive divergence and a horizontal flow directed from the center of the map outward. The time sequence of the continuum intensity in panel (d) reveals the recurrent appearance of exploding granules. These granules are marked by white arrows. Such families of repeatedly splitting granules can persist for long times (up to 8 hours, Müller et al. 2001; Roudier et al. 2003) and when averaged in time they give rise to positive divergences that are identified as mesogranules (Roudier \& Muller 2004).

In panel (b) a longitudinal magnetogram at $t=$
18.3 minutes is shown. The green contours trace the mesolanes and the red circles display the locations of sinks. Notice that in panel (a) the sinks are found at strong negative divergence areas in which the horizontal flows converge. In the magnetogram, the majority of the magnetic elements are located within the mesolanes and in particular inside or near sinks. As an example, in the bottom right sink two opposite polarity features are found and in the upper right one a prominent magnetic element is observed. The evolution of the latter was studied in detail in Requerey et al. (2015). It contains multiple inner magnetic cores which continuously fragment and merge in response to the evolution of the surrounding granules. Here, we find that this structure is indeed anchored to a persistent downdraft. This constant inward attraction may help maintain all the fragments together in a single magnetic structure. This is just one example of the processes occurring when a magnetic structure is located in a sink (see Section 4).

In the interior of the mesogranular cell, smaller magnetic patches are also observed. These features are much weaker, with a longitudinal magnetic field component smaller than $50 \mathrm{G}$. Some of them have been identified as small loop-like structures because a linear polarization patch appears flanked by two opposite polarities in the longitudinal magnetograms. We have tracked the evolution of their footpoints manually until they merge, cancel with other features, or fade away below the detection limit. We display their trajectories in panel (c). Blue and red lines stand for positive and negative polarity patches and crosses indicate their initial positions. Interestingly, they move radially toward the mesolanes, although they also describe random motions at smaller scales (cf. Abramenko et al. 2011; Manso Sainz et al. 2011; Jafarzadeh et al. 2014). In panel (d) we show the 


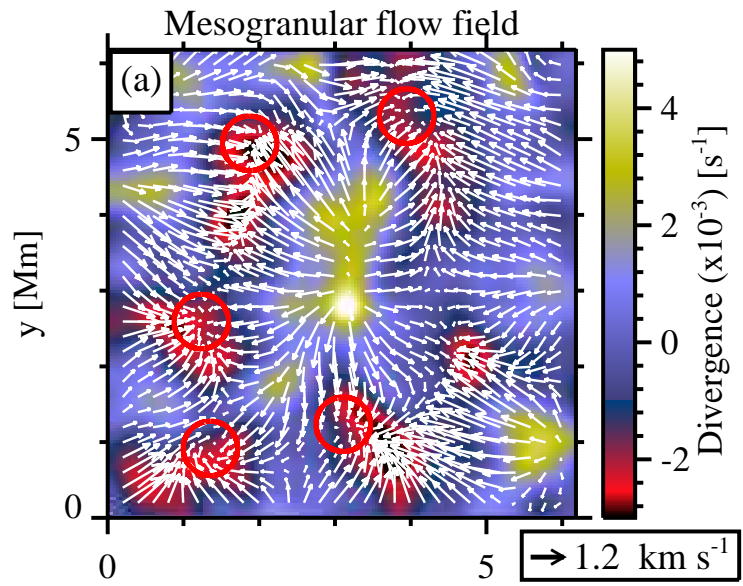

$\mathrm{x}[\mathrm{Mm}]$

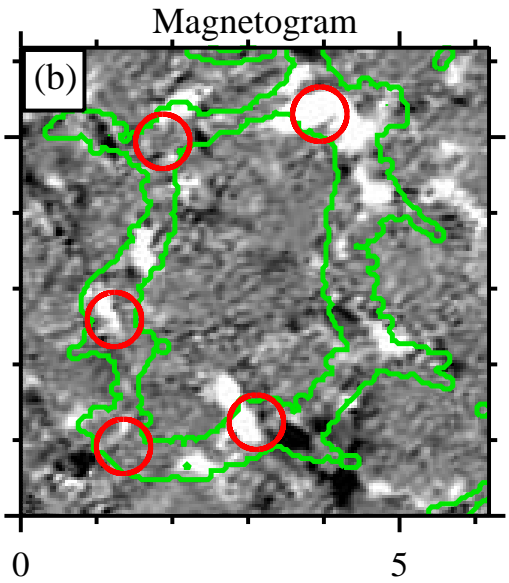

$\mathrm{x}[\mathrm{Mm}]$

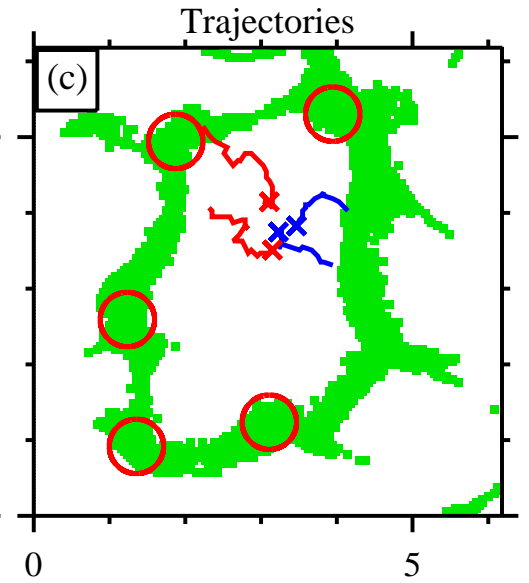

$\mathrm{x}[\mathrm{Mm}]$

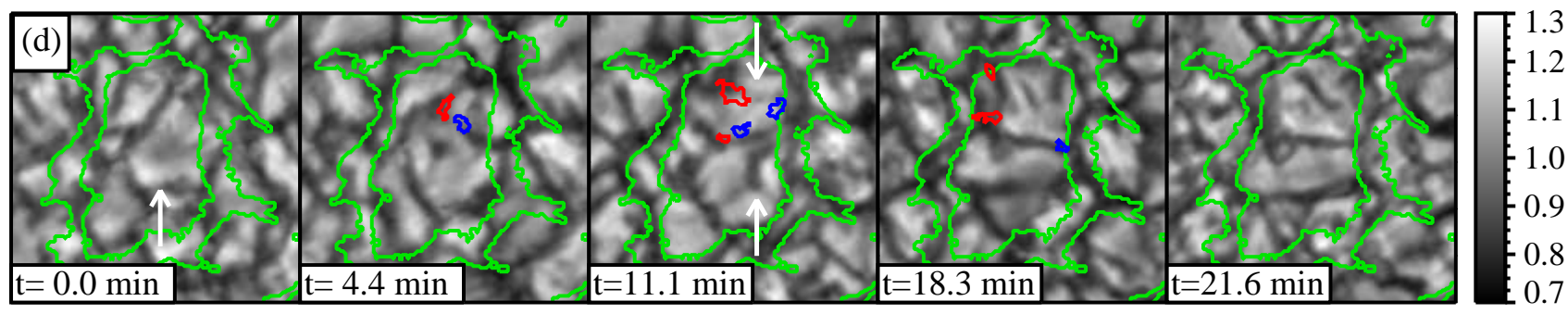

Figure 10. Closeup of a mesogranule. The area is located within the black solid rectangle in Figure 1. Panel (a): divergence map (background image) and mesogranular flow field (white arrows). Negative values represent convergence. Panel (b): longitudinal magnetogram at $t=18.3$ minutes saturated at $\pm 50 \mathrm{G}$. Green contours delineate the mesolanes. Panel (c): mesolanes (green pixels) and trajectories followed by the footpoints of initially detected small-scale magnetic loops. Blue and red paths refer to the positive and negative polarity magnetic patches, respectively. Crosses indicate the initial position of the footpoints. Red circles (with a radius of 9 pixel $\sim 360 \mathrm{~km}$ ) represent the positions of sinks. Panel (d): a time sequence of continuum intensity maps covering the same field of view. Arrows indicate exploding granules. Blue and red contours represent positive and negative polarity magnetic patches, respectively.

evolution of the patches overlaid on continuum intensity maps. Blue and red contours represent positive and negative polarity patches, respectively. They appear located at the edges of granules and are swept to the mesogranular lanes as the granules expand and explode. The uppermost negative polarity patch reaches the periphery of a sink where it soon cancels out with an opposite polarity feature. These two features are observed prior to their cancellation at the upper border of the top left sink in panel (b).

\section{DISCUSSION AND CONCLUSIONS}

The accuracy and high spatial resolution of the SuNRISE/IMaX polarimetric measurements have allowed us to quantify the relation between quiet-Sun magnetic fields, mesogranulation, and convectively driven sinks. We have computed the horizontal flow field through LCT of continuum intensity maps averaged over two time series of roughly 20 minutes each. From the inferred horizontal velocity vectors, we identified mesogranular lanes and convergence centers by tracking the evolution of Lagrange tracers. Converging flows are preferentially located at the junction of multiple meso- grangranular lanes. According to the cork trajectories and the distribution of converging flow fields we have found two types of converging flows, namely, (1) radial flows: radially symmetrical flow fields directed inwards to the convergence center; and (2) vortex flows: flow fields rotating around a vertical axis. We have detected 131 long-lived converging flows, $65 \%$ belonging to type 1 , and the rest to type 2 . This results in $6.7 \times 10^{-2}$ converging flows per $\mathrm{Mm}^{2}, 4.4 \times 10^{-2}$ radial flows per $\mathrm{Mm}^{2}$ and $2.4 \times 10^{-2}$ vortex flows per $\mathrm{Mm}^{2}$, respectively. The value for vortex flows is comparable to that found by Vargas Domínguez et al. (2011). It is important to remember that our LCT temporal average (21.6 minutes) and the tracking window $(600 \mathrm{~km})$ is almost identical to those used by Vargas Domínguez et al. (2011) of 20 minutes and $725 \mathrm{~km}$, respectively.

Unlike in previous works, here we have profited from the spectroscopic capabilities of IMaX to determine LOS velocities in converging flows. The LOS velocity histograms reveal that converging flows are (1) preferentially located within intergranular lanes and (2) tend to be associated with long-lived downdrafts. Accordingly, converging flows are better described as convec- 
tively driven sinks.

By studying individual examples, we have shown that sinks can affect the evolution of magnetic elements. In particular, coalescence, cancellation and fragmentation processes are seen to take place at localized downdrafts. We have also provided quantitative measurements of the relationship between magnetic fields, mesogranules and sinks. First, we have confirmed the finding of Yelles Chaouche et al. (2011) that the large majority $(80 \%)$ of magnetic fields with longitudinal component larger than $100 \mathrm{G}$ are located in mesogranular lanes. In addition, we have shown that the strongest magnetic features tend to concentrate in long-lived sinks, at the junction of mesogranules. Roughly $40 \%$ of the magnetic elements with longitudinal magnetic fields above $500 \mathrm{G}$ are found within $360 \mathrm{~km}$ of sink centers. In contrast, we found that $69.5 \%$ of the 400 small-scale magnetic loops detected by Martínez González et al. (2012) are located in mesogranules, $30.5 \%$ in mesolanes, and only $4.5 \%$ in the close neighborhood of long-lived sinks. These values are very similar to the surface fractions covered by each region and we conclude that magnetic loops are homogeneously distributed on mesogranular scales.

We have analyzed the evolution of two particular loops appearing inside a mesogranule. Their footpoints are passively advected by the mesogranular horizontal flows, as they are swept by exploding granules. Through this process they reach the boundaries of the underlying mesogranules and well localized sinks located at the vertices of mesogranular lanes. In such downdrafts they can be confined by converging granular flows and later concentrated up to kG values (Bushby et al. 2008; Danilovic et al. 2010b; Requerey et al. 2014). The advection of weak fields by mesogranular horizontal flows and their concentration in mesogranular vertices is also supported by magnetoconvection models that study the interaction of convective flows with an imposed field (see e.g., Stein \& Nordlund 2006). However, this scenario is also compatible with a small-scale dynamo (Rempel 2014). In fact, small-scale dynamo action is more efficient when mesogranulation is present (Bushby \& Favier 2014), and the coverage by $\mathrm{kG}$ fields doubles as a strong mesogranular network is formed (Rempel 2014).

Our results seem to reveal a hierarchical picture for the evolution of quiet Sun magnetic fields: flux emerges preferentially near the edges of granules in the form of small-scale magnetic loops (Centeno et al. 2007; Martínez González \& Bellot Rubio 2009; Danilovic et al. 2010a); the footpoints are first swept to nearby intergranular lanes unless they cancel out before (Centeno et al. 2007; Requerey et al. 2014), and then advected to mesogranular lanes, particularly to the junctions where long-lived downdrafts are formed. The concentration of weak fields in the mesogranular vertices gives rise to internetwork magnetic elements. If these magnetic elements are strong (long-lived) enough, supergranular flows can drag them along (de Wijn et al. 2008; Orozco Suárez et al. 2012), until they reach the supergranular boundaries and eventually contribute to the magnetic network flux (Gošić et al. 2014). Interestingly, this qualitative scenario may explain why most of the newly detected internetwork flux is not in the form of bipolar elements, but instead seems to be unipolar (Lamb et al. 2008; Gošić 2015; Anusha et al. 2016). Unipolar appearances are thought to be the result of the coalescence of previously unobservable, very weak flux into stronger features, as first pointed out by Lamb et al. (2008). The concentration of weak fields in sinks provides a plausible mechanism whereby this can occur.

We thank M. J. Martínez González for providing the locations of magnetic loops shown in Figure 1. We also thank the referee for constructive suggestions that contribute to improve the article. The work by I. S. R. has been funded by the Basque Government under a grant from Programa Predoctoral de Formación de Personal Investigador del Departamento de Educación, Universidades e Investigación. This work has been partially funded by the Spanish Ministerio de Economía y Competitividad, through Projects No. ESP2013-47349-C61-R and ESP2014-56169-C6-1-R, including a percentage from European FEDER funds. The German contribution has been funded by the Bundesministerium für Wirtschaft und Technologie through Deutsches Zentrum für Luft- und Raumfahrt e.V. (DLR), grant number 50 OU 0401, and by the Innovationsfond of the President of the Max Planck Society (MPG). This work was partly supported by the BK21 plus program through the National Research Foundation (NRF) funded by the Ministry of Education of Korea.

\section{REFERENCES}

Abramenko, V. I., Carbone, V., Yurchyshyn, V., et al. 2011, ApJ, 743, 133

Anusha, L. S., Solanki, S. K., Hirzberger, J., \& Feller, A. 2016, arXiv:1608.08499

Attie, R., Innes, D. E., \& Potts, H. E. 2009, A\&A, 493, L13
Balmaceda, L., Vargas Domínguez, S., Palacios, J., Cabello, I., \& Domingo, V. 2010, A\&A, 513, L6

Barthol, P., Gandorfer, A., Solanki, S. K., et al. 2011, SoPh, 268, 
Beck, C., Bellot Rubio, L. R., Schlichenmaier, R., Sütterlin, P. 2007, A\&A, 472, 607

Berkefeld, T., Schmidt, W., Soltau, D., et al. 2011, SoPh, 268, 103

Bonet, J. A., Márquez, I., Sánchez Almeida, J., Cabello, I., \& Domingo, V. 2008, ApJL, 687, L131

Bonet, J. A., Márquez, I., Sánchez Almeida, J., et al. 2010, ApJL, 723, L139

Brandt, P. N., Scharmer, G. B., Ferguson, S., Shine, R. A., \& Tarbell, T. D. 1988, Nature, 335, 238

Brandt, P. N., Ferguson, S., Shine, R. A., Tarbell, T. D., \& Scharmer, G. B. 1991, A\&A, 241, 219

Bushby, P. J., Houghton, S. M., Proctor, M. R. E., \& Weiss, N. O. 2008, MNRAS, 387, 698

Bushby, P. J., \& Favier, B. 2014, A\&A, 562, A72

Centeno, R., Socas-Navarro, H., Lites, B., et al. 2007, ApJL, 666, L137

Danilovic, S., Beeck, B., Pietarila, A., et al. 2010a, ApJL, 723, L149

Danilovic, S., Schüssler, M., \& Solanki, S. K. 2010b, A\&A, 509, A76

de Wijn, A. G., Lites, B. W., Berger, T. E., et al. 2008, ApJ, 684,1469

Gandorfer, A., Grauf, B., Barthol, P., et al. 2011, SoPh, 268, 35

Gingerich, O., Noyes, R. W., Kalkofen, W., \& Cuny, Y. 1971, SoPh, 18, 347

Gonsalves, R. A. 1982, Optical Engineering, 21, 829

Gošić, M., Bellot Rubio, L. R., Orozco Suárez, D., Katsukawa, Y., \& del Toro Iniesta, J. C. 2014, ApJ, 797, 49

Gošić, M. 2015, Ph.D. Thesis, Universidad de Granada

Jafarzadeh, S., Cameron, R. H., Solanki, S. K., et al. 2014, A\&A, 563, A101

Kitiashvili, I. N., Kosovichev, A. G., Wray, A. A., \& Mansour, N. N. 2010, ApJ, 719, 307

Kitiashvili, I. N., Kosovichev, A. G., Mansour, N. N., \& Wray, A. A. 2011, ApJL, 727, L50

Lamb, D. A., DeForest, C. E., Hagenaar, H. J., Parnell, C. E., \& Welsch, B. T. 2008, ApJ, 674, 520-529

Manso Sainz, R., Martínez González, M. J., \& Asensio Ramos, A. 2011, A\&A, 531, L9

Martínez González, M. J., Collados, M., Ruiz Cobo, B., \& Solanki, S. K. 2007, A\&A, 469, L39

Martínez González, M. J., \& Bellot Rubio, L. R. 2009, ApJ, 700, 1391

Martínez González, M. J., Manso Sainz, R., Asensio Ramos, A., \& Bellot Rubio, L. R. 2010, ApJL, 714, L94

Martínez González, M. J., Manso Sainz, R., Asensio Ramos, A., \& Hijano, E. 2012, ApJ, 755, 175

Martínez Pillet, V., Del Toro Iniesta, J. C., Álvarez-Herrero, A., et al. 2011, SoPh, 268, 57

Moll, R., Cameron, R. H., \& Schüssler, M. 2011, A\&A, 533, A126

Molowny-Horas, R., \& Yi, Z. 1994, Internal Rep. 31, Institute of Theoretical Astrophysics (Oslo: Univ. Oslo)

Muller, R., Auffret, H., Roudier, T., et al. 1992, Nature, 356, 322

Müller, D. A. N., Steiner, O., Schlichenmaier, R., \& Brandt, P. N. 2001, SoPh, 203, 211

Nordlund, A. 1985, SoPh, 100, 209

Nordlund, Å., Stein, R. F., \& Asplund, M. 2009, Living Reviews in Solar Physics, 6,
November, L. J., Toomre, J., Gebbie, K. B., \& Simon, G. W. 1981, ApJL, 245, L123

November, L. J., \& Simon, G. W. 1988, ApJ, 333, 427

Orozco Suárez, D., Katsukawa, Y., \& Bellot Rubio, L. R. 2012, ApJL, 758, L38

Park, S.-H., Tsiropoula, G., Kontogiannis, I., et al. 2016, A\&A, 586, A25

Parker, E. N. 1978, ApJ, 221, 368

Paxman, R. G., Seldin, J. H., Loefdahl, M. G., Scharmer, G. B., \& Keller, C. U. 1996, ApJ, 466, 1087

Pötzi, W., \& Brandt, P. N. 2005, Hvar Observatory Bulletin, 29, 61

Pötzi, W., \& Brandt, P. N. 2007, Central European

Astrophysical Bulletin, 31, 11

Rempel, M. 2014, ApJ, 789, 132

Requerey, I. S., Del Toro Iniesta, J. C., Bellot Rubio, L. R., et al. 2014, ApJ, 789, 6

Requerey, I. S., Del Toro Iniesta, J. C., Bellot Rubio, L. R., et al. 2015, ApJ, 810, 79

Riethmüller, T. L., Solanki, S. K., Berdyugina, S. V., et al. 2014, A\&A, 568, A13

Roudier, T., Malherbe, J. M., Vigneau, J., \& Pfeiffer, B. 1998, A\&A, 330, 1136

Roudier, T., Lignières, F., Rieutord, M., Brandt, P. N., \& Malherbe, J. M. 2003, A\&A, 409, 299

Roudier, T., \& Muller, R. 2004, A\&A, 419, 757

Ruiz Cobo, B., \& del Toro Iniesta, J. C. 1992, ApJ, 398, 375

Shelyag, S., Keys, P., Mathioudakis, M., \& Keenan, F. P. 2011, A\&A, 526, A5

Simon, G. W., Title, A. M., Topka, K. P., et al. 1988, ApJ, 327, 964

Solanki, S. K., Barthol, P., Danilovic, S., et al. 2010, ApJL, 723, L127

Spruit, H. C. 1976, SoPh, 50, 269

Spruit, H. C. 1979, SoPh, 61, 363

Spruit, H. C., \& Zweibel, E. G. 1979, SoPh, 62, 15

Spruit, H. C., Nordlund, A., \& Title, A. M. 1990, ARA\&A, 28, 263

Stein, R. F., \& Nordlund, Å. 1998, ApJ, 499, 914

Stein, R. F., \& Nordlund, Å. 2006, ApJ, 642, 1246

Steiner, O., \& Rezaei, R. 2012, Fifth Hinode Science Meeting, 456,3

Title, A. M., Tarbell, T. D., Topka, K. P., et al. 1989, ApJ, 336, 475

Vargas Domínguez, S., Palacios, J., Balmaceda, L., Cabello, I., \& Domingo, V. 2011, MNRAS, 416, 148

Vargas Domínguez, S., Palacios, J., Balmaceda, L., Cabello, I., \& Domingo, V. 2015, SoPh, 290, 301

Wang, Y., Noyes, R. W., Tarbell, T. D., \& Title, A. M. 1995, ApJ, 447, 419

Webb, A. R., \& Roberts, B. 1978, SoPh, 59, 249

Wedemeyer-Böhm, S., \& Rouppe van der Voort, L. 2009, A\&A, 507, L9

Wedemeyer-Böhm, S., Scullion, E., Steiner, O., et al. 2012, Nature, 486, 505

Yelles Chaouche, L., Moreno-Insertis, F., Martínez Pillet, V., et al. 2011, ApJL, 727, L30

Yi, Z. 1992, PhD thesis, University of Oslo, Oslo 